\title{
Spirals and ribbons in counter-rotating Taylor-Couette flow: frequencies from mean flows and heteroclinic orbits
}

\author{
Yacine Bengana and Laurette S. Tuckerman \\ Laboratoire de Physique et Mécanique des Milieux Hétérogènes (PMMH), CNRS, ESPCI Paris, \\ PSL Research University; Sorbonne Université, Univ. Paris Diderot, 75005 Paris France
}

Physical Review Fluids 4, 044402 (2019)

\begin{abstract}
A number of time-periodic flows have been found to have a property called RZIF: when a linear stability analysis is carried out about the temporal mean (rather than the usual steady state), an eigenvalue is obtained whose Real part is Zero and whose Imaginary part is the nonlinear Frequency. For two-dimensional thermosolutal convection, a Hopf bifurcation leads to traveling waves which satisfy the RZIF property and standing waves which do not. We have investigated this property numerically for counter-rotating Couette-Taylor flow, in which a Hopf bifurcation gives rise to branches of upwards and downwards traveling spirals and ribbons which are an equal superposition of the two. In the regime that we have studied, we find that both spirals and ribbons satisfy the RZIF property. As the outer Reynolds number is increased, the ribbon branch is succeeded by two types of heteroclinic orbits, both of which connect saddle states containing two axially stacked pairs of axisymmetric vortices. One heteroclinic orbit is non-axisymmetric, with excursions that resemble the ribbons, while the other remains axisymmetric.
\end{abstract}

PACS numbers: $47.20 . \mathrm{Ky}, 47.20 . \mathrm{Qr}$ 


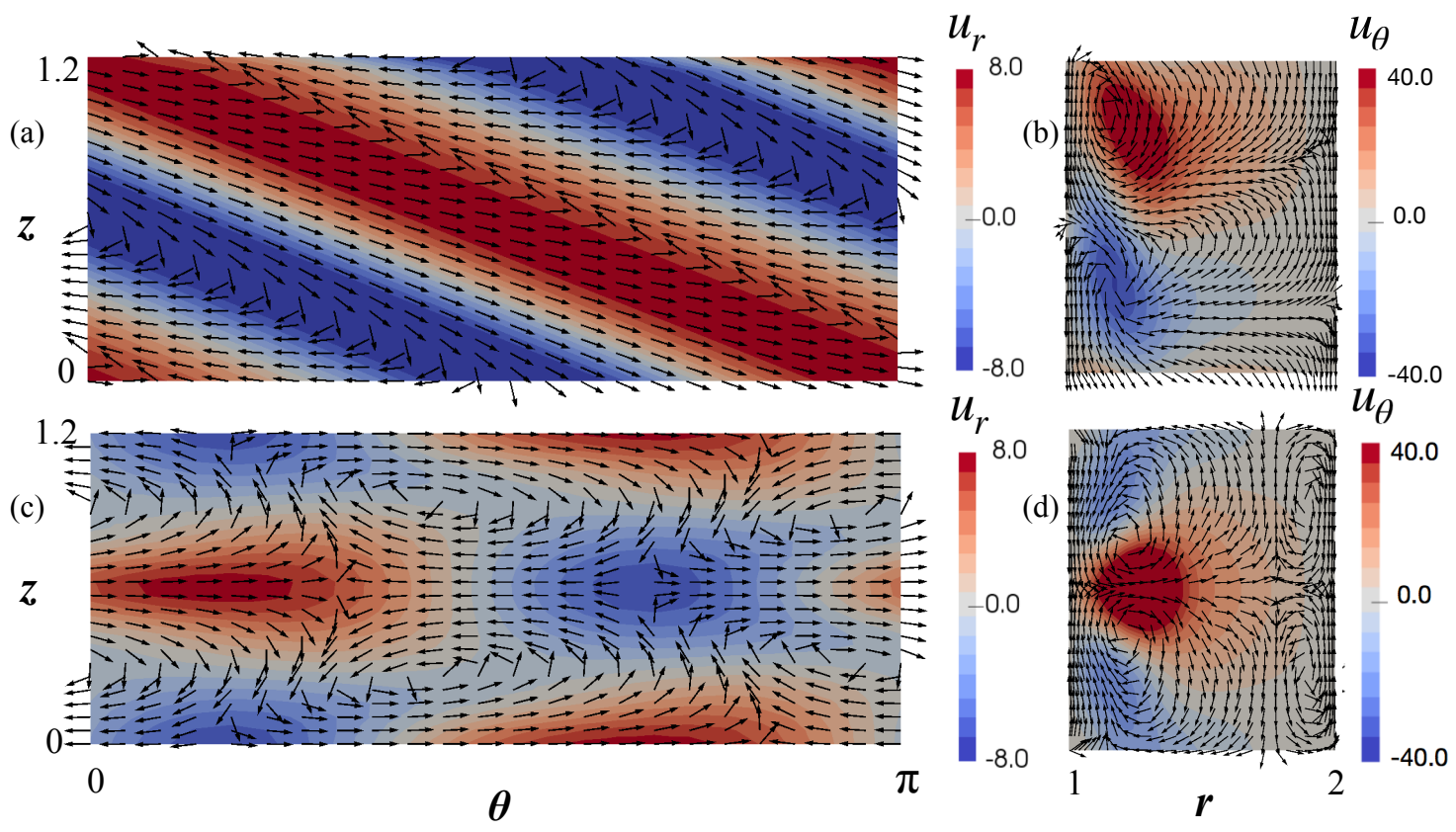

FIG. 1. Nonlinear spirals $(\mathrm{a}, \mathrm{b})$ and ribbons $(\mathrm{c}, \mathrm{d})$ at $R e=-550$. The deviation from laminar Couette flow is shown. Spirals of the opposite helicity exist and have identical properties but are not shown. a,c) $(\theta, z)$ velocity (arrows) with $u_{r}$ (colors) at $r=1.26$, near the maximum of the deviation from laminar Couette flow. b,d) $(r, z)$ velocity (arrows) with $u_{\theta}$ (colors).

\section{INTRODUCTION}

A number of time-periodic flows have been found to have the following property: when a linear stability analysis is carried out about the temporal mean (rather than the usual steady state from which they bifurcate), an eigenvalue emerges whose imaginary part reproduces the frequency of the nonlinear oscillation and whose real part is near zero. This property was first discovered by Barkley [1] and extensively studied [2 7] for the cylinder wake (for which it is strongly verified) and then for an open cavity [7 9] (for which it holds quite well, though not exactly). In these two-dimensional flows, a horizontal velocity imposed at infinity is deviated as it encounters objects (a cylinder) or openings (a cavity) and, for sufficiently high Reynolds number, undergoes a Hopf bifurcation.

In Turton, Tuckerman \& Barkley [10, this property was named RZIF - a mnemonic for the fact that the eigenvalue obtained by linearizing about the mean flow has a Real part of Zero and an Imaginary part which is the nonlinear Frequency - and was studied for a quite different kind of flow. Thermosolutal convection is driven by a vertical density gradient in a fluid mixture between two horizontal plates, which in turn is caused by imposed temperature and concentration differences at the two bounding plates. When the temperature and concentration have opposing effects on the density, instability is manifested as a Hopf bifurcation. In a two-dimensional domain with horizontally periodic boundary conditions, this is a classic case of the breaking of $O(2)$ symmetry (translations and reflection) and leads to the simultaneous formation of traveling and standing wave branches, at most one of which can be stable [11]. Turton et al. [10] discovered that the traveling waves in thermosolutal convection provide another clear realisation of the RZIF property, while the standing waves provide an equally clear counterexample: the real part of the eigenvalue obtained by linearizing about the mean part of the standing waves is far from zero and its imaginary part is far from the nonlinear frequency. 
We have sought to continue this investigation on another pair of hydrodynamic traveling and standing waves. In Taylor-Couette flow, when only the inner cylinder rotates, a steady bifurcation leads to the classic Taylor vortices. In counter-rotating Taylor-Couette flow, however, these vortices are inclined to the rotation axis, and hence nonaxisymmetric. A Hopf bifurcation then gives rise to branches of the well-known spirals [12 14] and the somewhat lesser-known ribbons [15 23], which, like standing waves, are an equal superposition of spirals moving axially upwards and downwards. As in [10, 11, this is a consequence of the breaking of $O(2)$ symmetry, here in the axial direction, when end effects are disregarded. Figure 1 shows the appearance of these two types of flows.

Counter-rotating Taylor-Couette flow has an additional symmetry when compared to the two-dimensional thermosolutal cases studied in [10, 11. Along with the $O(2)$ axial $z$ symmetry, the Taylor-Couette configuration has $S O(2)$ symmetry, consisting of rotations (not reflection) in the azimuthal $\theta$ direction. The tilted spirals or azimuthally tiled ribbons are not axisymmetric, and hence they break $S O(2)$ symmetry. In such cases, bifurcations always lead to states which rotate in the azimuthal direction; see e.g. 24. Thus, although ribbons are standing waves in the axial direction, i.e. equal superpositions of axially-upwards and axially-downwards traveling waves, they rotate in the azimuthal direction, as do the spirals.

As the rotation velocity of the outer cylinder is varied, the ribbon branch is succeeded by two types of heteroclinic orbits, both of which connect saddle states consisting of two pairs of axisymmetric vortices. One heteroclinic orbit is non-axisymmetric, with excursions that resemble the ribbons, while the other remains axisymmetric.

\section{METHODS}

To investigate this problem numerically, we have used the spectral finite-difference code of Willis [25] to solve the Navier-Stokes equations in a cylindrical annulus,

$$
\begin{aligned}
\partial_{t} \mathbf{U} & =-(\mathbf{U} \cdot \nabla) \mathbf{U}-\nabla P+\nabla^{2} \mathbf{U} \\
\nabla \cdot \mathbf{U} & =0 \\
\mathbf{U} & =\mathbf{e}_{\theta} R e_{\mathrm{in}}, \mathbf{e}_{\theta} R e_{\mathrm{out}} \quad \text { at } r_{\mathrm{in}}, r_{\mathrm{out}}
\end{aligned}
$$

where the length scale is $d \equiv r_{\text {out }}-r_{\text {in }}$ and the time scale is $d^{2} / \nu$, where $\nu$ is the kinematic viscosity. The radius ratio is $\eta \equiv r_{\text {in }} / r_{\text {out }}$, the two Reynolds numbers are defined as $R e_{j} \equiv r_{j} \Omega_{j} d / \nu$ where $j=$ in, out, and the numerical resolution is $\left(N_{r}, N_{\theta}, N_{z}\right)=(33,16,16)$.

We use the parameters in Pinter et al. [18 20], namely radius ratio $\eta \equiv r_{\text {in }} / r_{\text {out }}=0.5$, inner Reynolds number $R e_{\text {in }}=$ 240 and azimuthal wavenumber $M_{0}=2$ (azimuthal wavelength $2 \pi / M_{0}=\pi$ ). We have chosen the axial wavelength $\lambda_{z}=1.2$, slightly less than the value studied intensively by [18 20 to avoid a secondary bifurcation they describe near the onset of spirals and ribbons for $\lambda_{z}=1.3$. Because counter-rotating Taylor-Couette flow is centrifugally unstable only near the inner cylinder, here for $r \in[1,1.24]$, the preferred axial wavelength is correspondingly less than that which would be expected if the vortices filled the gap. Because $R e_{\text {in }}$ remains fixed and we will vary $R e_{\text {out }}$, we will simplify the notation by using $R e$ instead of $R e_{\text {out }}$ to designate the outer Reynolds number.

We have modified the Taylor-Couette code [25] in order to timestep the equations linearized about a velocity field $\mathbf{U}$ :

$$
\begin{aligned}
\partial_{t} \mathbf{u} & =-(\mathbf{U} \cdot \nabla) \mathbf{u}-(\mathbf{u} \cdot \nabla) \mathbf{U}-\nabla p+\nabla^{2} \mathbf{u} \\
\nabla \cdot \mathbf{u} & =0 \\
\mathbf{u} & =0 \quad \text { at } r_{\text {in }}, r_{\text {out }}
\end{aligned}
$$

Time-integration of the linearized equations (2) about a steady solution $\mathbf{U}$ converges to the leading eigenvector, i.e. that whose corresponding eigenvalue has the most positive or least negative real part or growth rate. It is a means of carrying out the power method on the exponential of the right-hand-side of (2). Integration of (2) does not generally lead to a steady or periodic state, since the nonlinear terms which would otherwise saturate the amplitude is absent. Instead, it leads to states whose amplitude increases or decreases, but with a constant growth rate and frequency, in the case of a complex eigenvalue.

We first use the linearized code to compute the Hopf bifurcation threshold from laminar Couette flow. For various timesteps $\Delta t$, we calculate the growth rate $\sigma$ as a function of $R e$ and then use linear extrapolation or interpolation to find $R e_{c}$ such that $\sigma\left(R e_{c}\right)=0$. (This procedure circumvents critical slowing down and so finds bifurcation thresholds faster and more accurately than could be done with nonlinear simulations.) The bifurcation thresholds calculated using $\Delta t=10^{-4}, 10^{-5}$, and $10^{-6}$ are, respectively, $R e_{c}=-595.23, R e_{c}=-586.65, R e_{c}=-585.42$, the last of which we consider to be the converged value. (For greater legibility and simplicity, in what follows we cite its truncated value -585 .) 
(a)

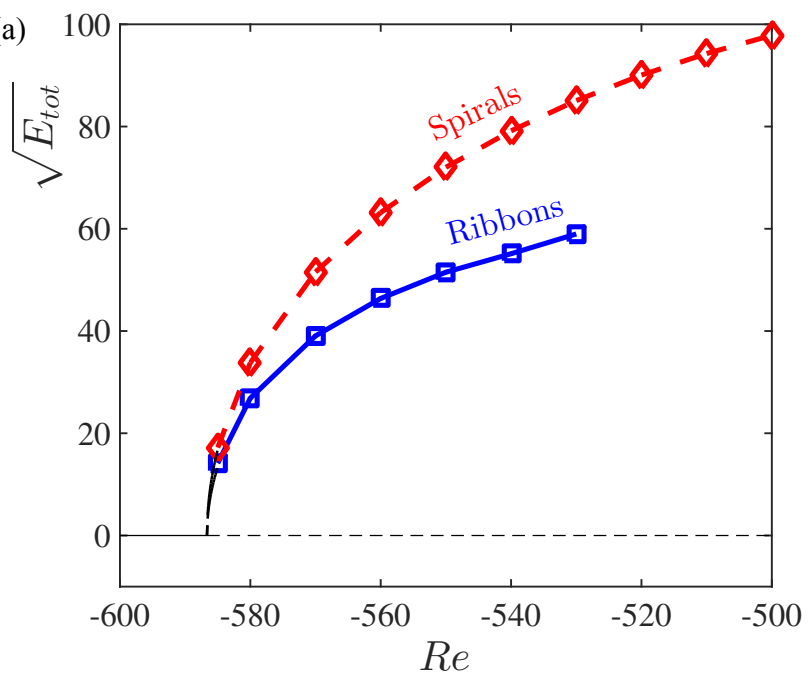

(b)

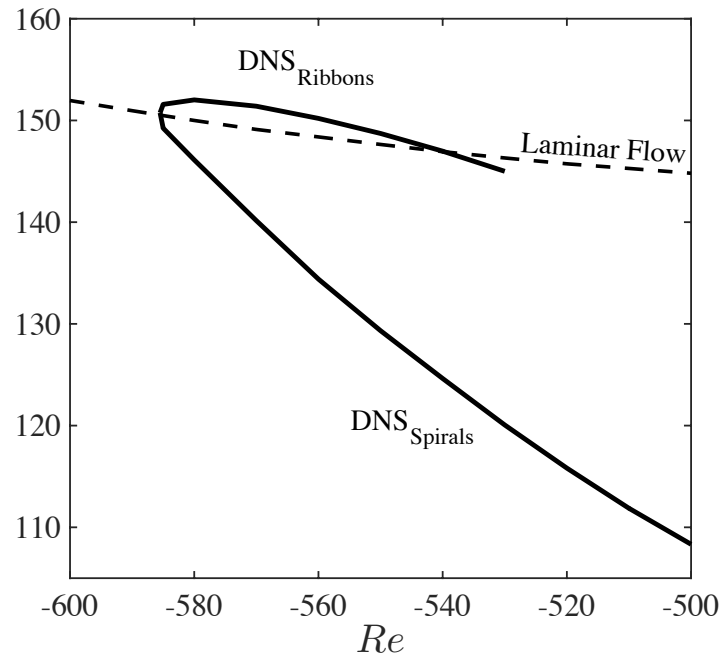

FIG. 2. a) Bifurcation diagram for spirals and ribbons. Square root of kinetic energy of the deviation from laminar Couette flow is shown as a function of $R e$. The ribbons are stable where shown, while the spirals are calculated by using a spiral initial condition. b) Frequencies of spiral and ribbon states as a function of Re. The dashed line shows the eigenvalues obtained by linearizing about laminar Couette flow.

The branches of spirals and ribbons illustrated in figure 1 bifurcate at $R e \approx-585$ towards increasing $R e$. Their functional form is:

$$
\begin{aligned}
& u^{\mathrm{SPI} \pm}(r, \theta, z, t)=\sum_{m} \hat{\mathbf{u}}_{m}(r) e^{i m\left(z / \lambda_{z} \pm M_{0} \theta-\omega_{\mathrm{SPI}} t\right)} \\
& u^{\mathrm{RIB}}(r, \theta, z, t)=\sum_{k, m}\left\{\begin{array}{ll}
\hat{u}_{k m, r}(r) \mathbf{e}_{r} & \sin \left(k z / \lambda_{z}\right) \\
\hat{u}_{k m, \theta}(r) \mathbf{e}_{\theta} & \sin \left(k z / \lambda_{z}\right) \\
\hat{u}_{k m, z}(r) \mathbf{e}_{z} & \cos \left(k z / \lambda_{z}\right)
\end{array}\right\} e^{i m\left(M_{0} \theta-\omega_{\mathrm{RIB}} t\right)}
\end{aligned}
$$

The spirals are traveling waves: they depend on $z, \theta$, and $t$ only via the single combination $z / \lambda_{z}+M_{0} \theta-\omega_{\mathrm{SPI}} t$. The ribbons have fixed axial nodal lines and are reflection symmetric; the axis of symmetry is $z=\lambda_{z} / 2$ in equation $(3)$ and in figure 1 . The ribbons are traveling waves in $\theta$ : they depend on $\theta$ and $t$ via $M_{0} \theta-\omega_{\text {RIB }} t$. Many other complex and esthetic symmetry-breaking flows have been observed in numerical simulations of this configuration [18, 23], including cross spirals, oscillating cross spirals, mixed cross spirals and mixed ribbons.

We will use numerical simulations of the nonlinear and linearized equations (1) and (2) to investigate the RZIF phenomenon mentioned in the introduction, in which linearization about the mean flow of a periodic solution yields an eigenvalue whose Real part is Zero and whose Imaginary part is the nonlinear Frequency. To do so, we will calculate the spirals and ribbons by time integrating the nonlinear equations (1) until an asymptotic state is reached. Because the spirals and ribbons are both azimuthally traveling waves, their temporal means are identical with their azimuthal means which are, in turn, the axisymmetric $(m=0)$ components of $(3)$. We will then find the eigenvalues and eigenvectors about the resulting mean flows by integrating equations (2).

\section{RZIF ANALYSIS}

We begin by showing the bifurcation diagram for spirals and ribbons. Their common threshold is $R e \approx-585$. On figure $2 \mathrm{a}$, we represent the square root of kinetic energy of the two branches. In this parameter range, the spirals are unstable to ribbons, but can be computed by including only $k=m$ modes or, like [18], by setting the $m=0$ component of $u_{r}$ to zero. The ribbons themselves become unstable for $R e \gtrsim-530$. Figure $2 \mathrm{~b}$ shows the frequency of the nonlinear spirals and ribbons, as well as those obtained by linearizing about laminar Couette flow (dashed line). Linearization about the laminar flow leads to the same set of eigenvalues for spirals or ribbons, since the eigenvectors corresponding to upgoing and downgoing spirals are related by symmetry, with their superposition leading to ribbons. For a supercritical Hopf bifurcation, these necessarily match the frequency of the nonlinear branches at the threshold. As $R e$ is increased, the frequencies of the nonlinear spirals deviate greatly from those derived from the laminar flow, but the frequencies of the nonlinear ribbons remain close. This was also true of the standing waves of thermosolutal 
(a)

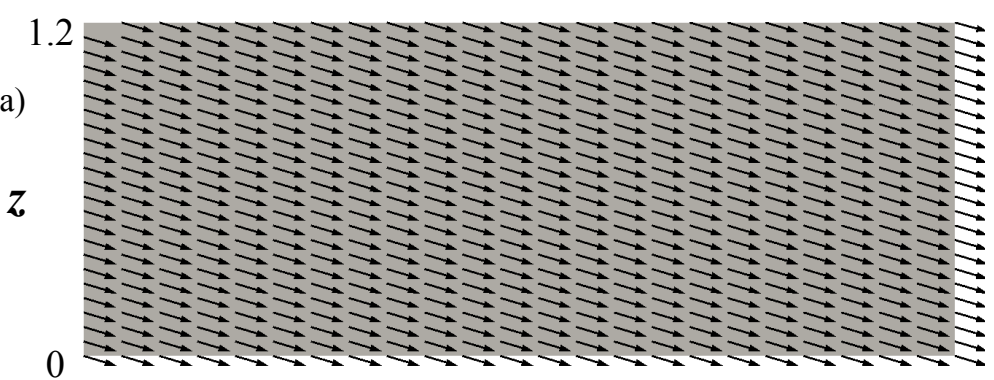

(c)

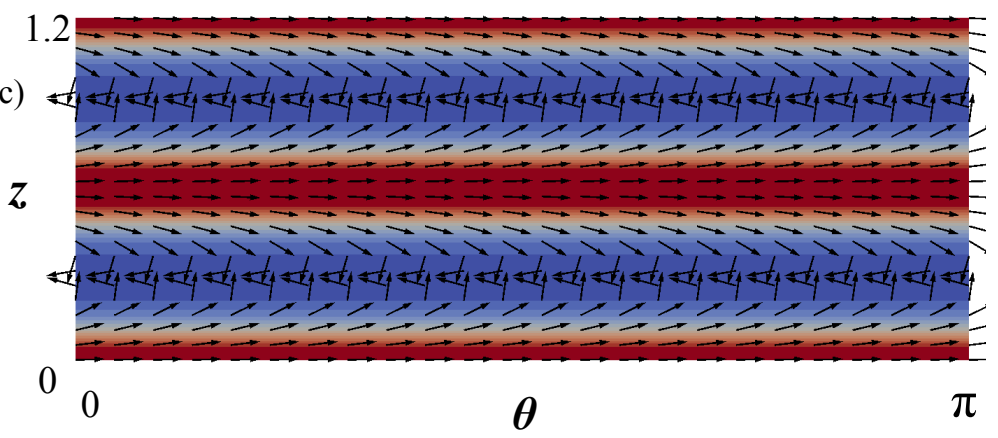

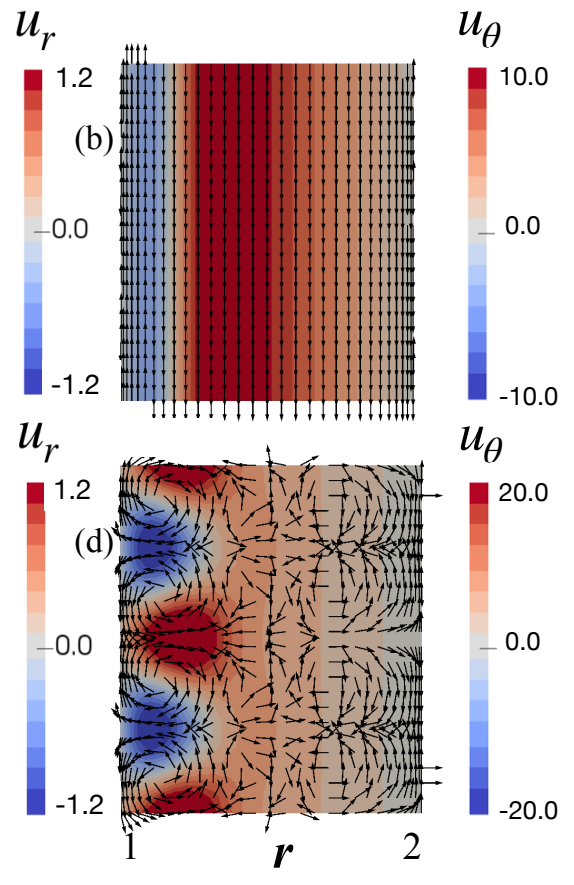

FIG. 3. Mean flow of spirals $(\mathrm{a}, \mathrm{b})$ and ribbons $(\mathrm{c}, \mathrm{d})$ at $R e=-550$. The deviation from laminar Couette flow is shown. a,c) $(\theta, z)$ velocity (arrows) with $u_{r}$ (colors) at $r=1.26$, near the maximum of the deviation from laminar Couette flow. b,d) $(r, z)$ velocity (arrows) with $u_{\theta}$ (colors). Since the spirals are traveling waves in the $\theta$ and $z$ directions, their means have no dependence on either. The ribbons are traveling waves in $\theta$ and hence their mean is axisymmetric.
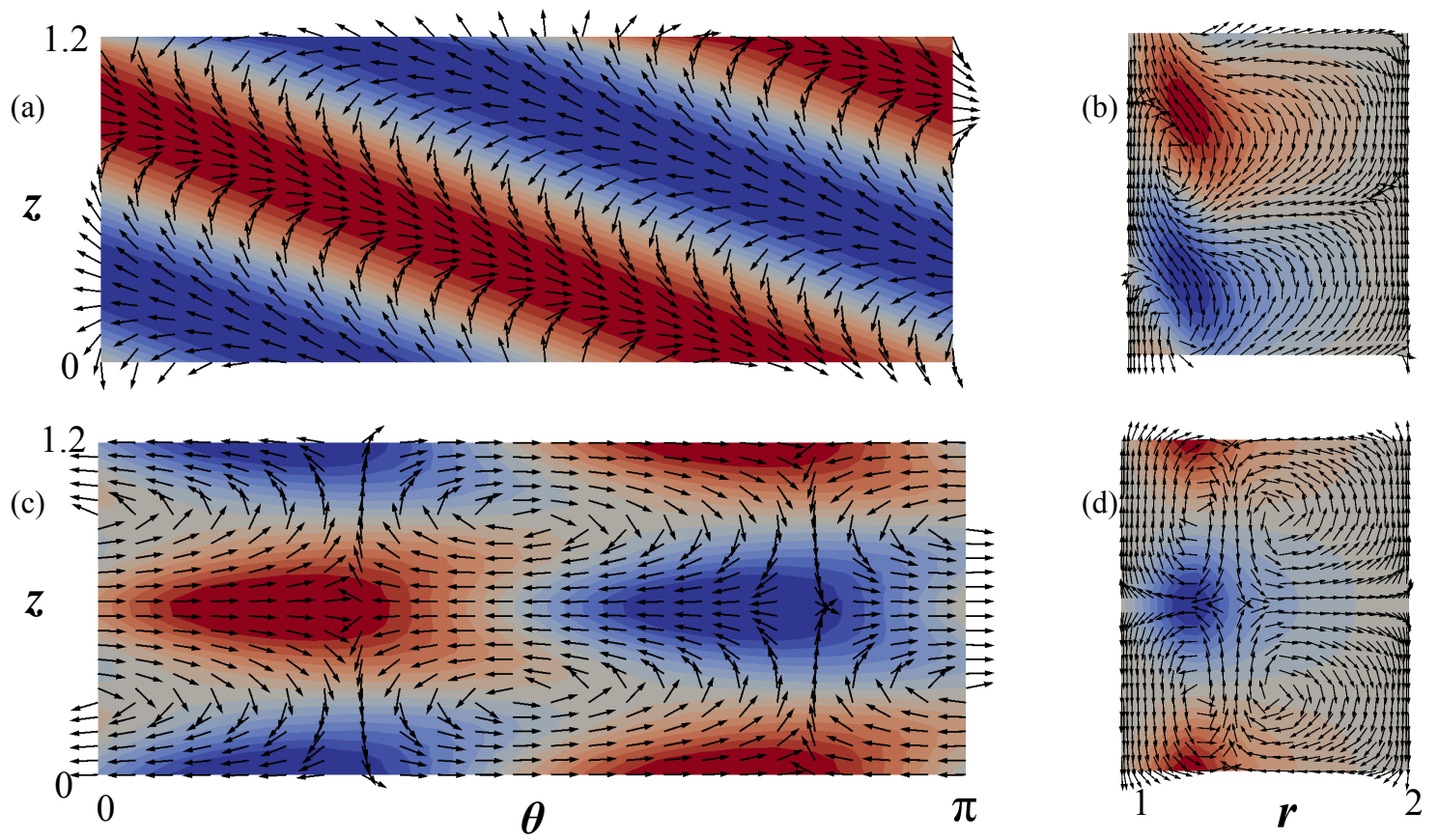

FIG. 4. Eigenvectors resulting from linearization about mean flow of spirals (a,b) and ribbons (c,d) at $R e=-550$. a) $(\theta, z)$ velocity (arrows) with $u_{r}$ (colors) at $r=1.26$. b) $(r, z)$ velocity (arrows) with $u_{\theta}$ (colors). These eigenvectors resemble the deviation of nonlinear spirals and ribbons from the laminar Couette flow as well as the eigenvectors of spiral and ribbon form obtained from linearizing about the laminar flow. 

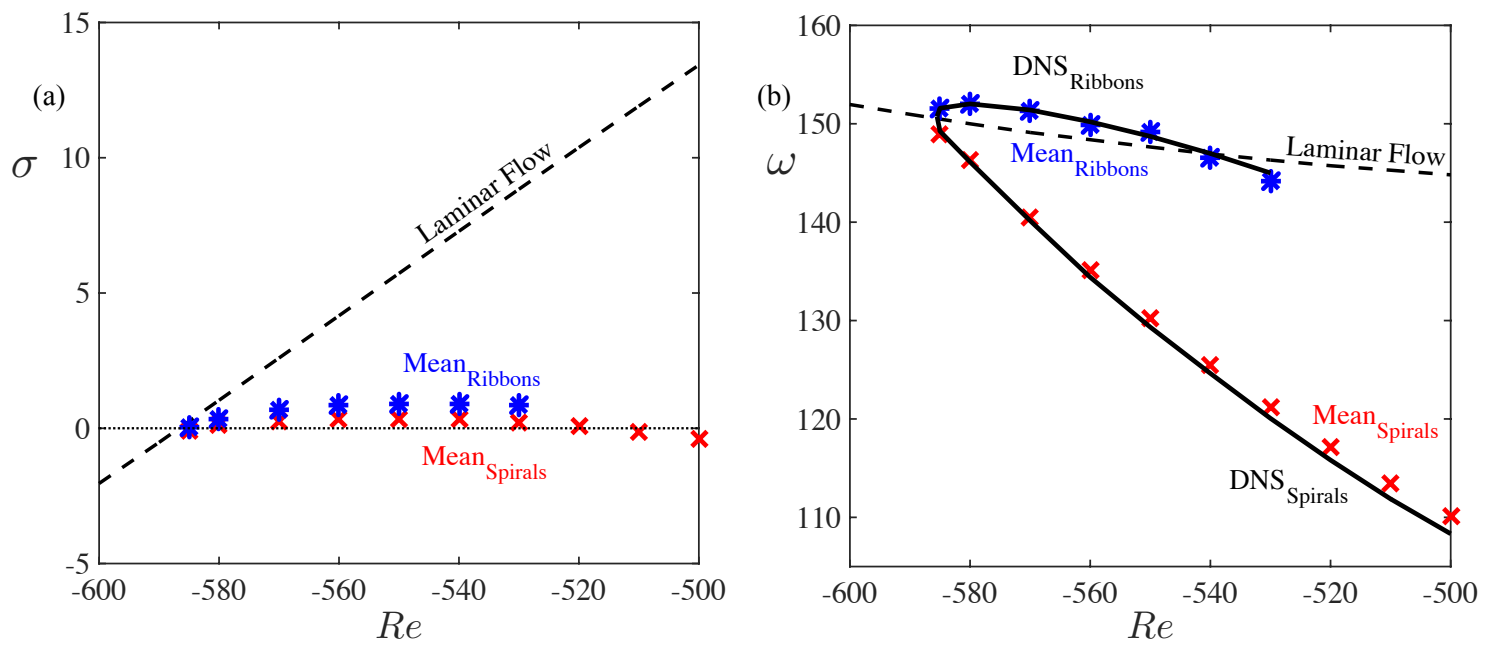

FIG. 5. Growth rates (a) and frequencies (b). Results from nonlinear numerical simulations (black curves) and from NavierStokes equations linearized about the laminar flow (dashed lines), about the mean flow of spirals (red crosses), and about the mean flow of ribbons (blue stars). Satisfaction of the RZIF property for the frequencies is demonstrated by the agreement between the red crosses and the black curve for spirals and between the blue stars and the black curve for ribbons. Satisfaction of the RZIF property for the growth rates is demonstrated by the fact that the blue stars and the red crosses are near zero.
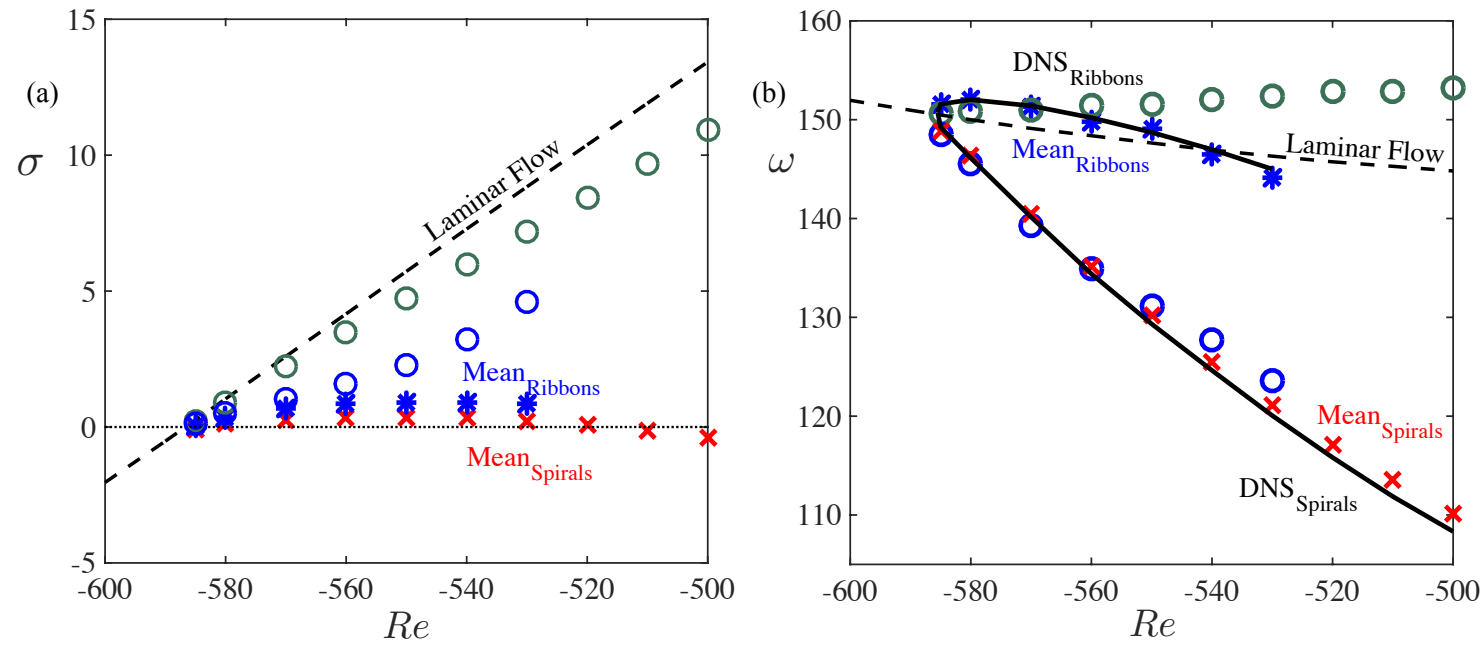

FIG. 6. Same as figure 5 with additional eigenvalues. The hollow circles are eigenvalues corresponding to spiral eigenvectors. The dark green circles (upper set) are obtained by linearizing around a mean spiral flow whose orientation is opposite to that of the eigenvector while the blue ones (lower set) are obtained by linearizing around the mean flow of a ribbon.

convection [10, which inspired this study: the frequencies of the nonlinear standing waves were much closer to those obtained by linearization about the laminar (conductive) flow than were the frequencies of the nonlinear traveling waves.

Figure 3 shows the temporal means of the nonlinear spiral and ribbon states shown in figure 1 at $R e=-550$. Since spirals are traveling waves in both the $\theta$ and the $z$ direction, their temporal means are equivalent to their spatial means and have no dependence on either direction. Ribbons are traveling waves in the $\theta$ direction and so their means have no $\theta$ dependence. We set $\mathbf{U}$ in the linearized equations (2) to the mean flows of spirals or ribbons shown in figure 3 and initialize $\mathbf{u}$ with fields of the spiral or ribbon form, respectively. We then integrate until the growth rate and the frequency are constant. The resulting field $\mathbf{u}(t)$ then cycles between the real and imaginary parts of the eigenvector which here are related by a spatial shift. Figure 4 shows samples of these eigenvectors. These greatly resemble the deviations of the corresponding nonlinear flows from laminar Couette flow shown in figure 1 and resemble even more the usual eigenvectors of spiral and ribbon form resulting from linearization about laminar Couette flow. 

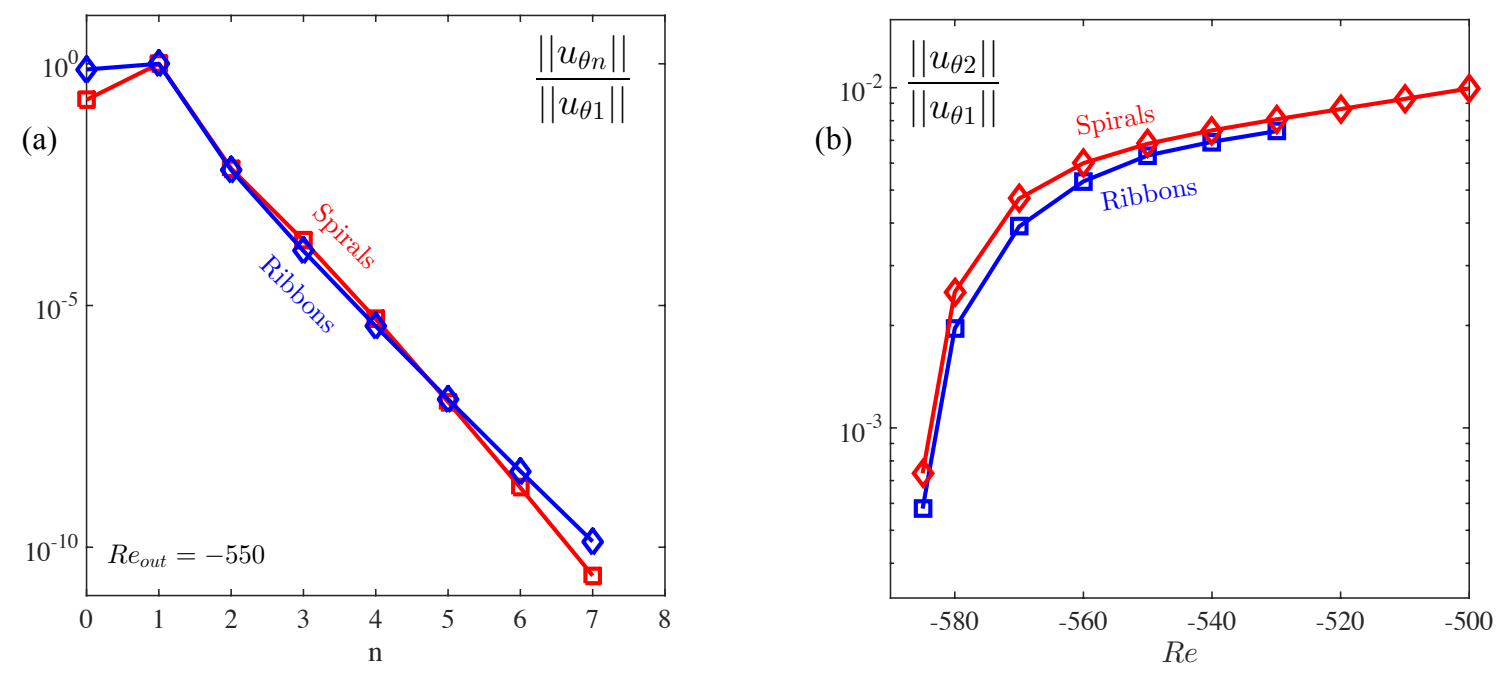

FIG. 7. Spectra of spirals and ribbon states. a) Full spectrum for states at $R e=-550$, normalized by amplitude of fundamental frequency. b) Ratio of second harmonic to fundamental frequency.

Figure 5 presents the main result of this section, namely that both the spirals and the ribbons satisfy the RZIF property. The growth rates or real parts of eigenvalues are shown in figure 5 a and the frequencies or imaginary parts are shown in figure 5b. The solid curves show the frequencies obtained from nonlinear simulations. Linearization about the mean flows of spirals (red crosses) or of ribbons (blue stars) lead to eigenvalues whose imaginary parts agree with the nonlinear frequencies. The corresponding growth rates are seen to be near zero, i.e. the mean flows are nearly marginally stable. (This has no relation to the marginal stability of the full spirals or ribbons to shifted versions of themselves, which arises from their $\theta$ or $z$ dependence in a homogeneous domain.) Thus, both the spirals and the ribbons satisfy the RZIF property. This contrasts with the thermosolutal case, in which the traveling waves satisfied the RZIF property, while the standing waves did not.

Additional eigenvalues are shown in figure 6, which are not related to the basic RZIF property. Both correspond to spiral eigenvectors and have positive growth rates. These show the frequency and growth rate of spiral eigenvectors superposed on the mean flow of spirals of opposite helicity (dark green circles) or of ribbons (blue circle). The positive growth rate for the spirals of opposite chirality could be related to the linear instability of the spirals to ribbons; such a transition would take place via the addition of spirals of opposite chirality until equal amplitudes are reached. However, for these parameter values, ribbons show no tendency to make a transition to spirals, and yet the growth rates from the mean flow of ribbons to spirals are also positive (although less so than the growth rates from the mean flow of spirals). More understanding of the meaning of linearization about mean flows would be necessary to interpret these eigenvalues.

The RZIF property is implied by a near-monochromatic spectrum, as shown in [10] as follows. Consider the evolution equation

$$
\partial_{t} \mathbf{U}=\mathcal{L} \mathbf{U}+\mathcal{N}(\mathbf{U}, \mathbf{U})
$$

where $\mathcal{L}$ is linear and $\mathcal{N}(\cdot, \cdot)$ is a quadratic nonlinearity. Let

$$
\mathbf{U}=\overline{\mathbf{U}}+\sum_{n \neq 0} \mathbf{u}_{n} e^{i n \omega t}
$$

(with $\mathbf{u}_{-n}=\mathbf{u}_{n}^{*}$ ) be the temporal Fourier decomposition of a periodic solution to (4) with mean $\overline{\mathbf{U}}$ and frequency $\omega$. The $n=1$ component of 4 is

$$
i \omega \mathbf{u}_{1}=\underbrace{\mathcal{L} \mathbf{u}_{1}+\mathcal{N}\left(\overline{\mathbf{U}}, \mathbf{u}_{1}\right)+\mathcal{N}\left(\mathbf{u}_{1}, \overline{\mathbf{U}}\right)}_{\mathcal{L}_{\bar{U}} \mathbf{u}_{1}}+\underbrace{\mathcal{N}\left(\mathbf{u}_{2}, \mathbf{u}_{-1}\right)+\mathcal{N}\left(\mathbf{u}_{-1}, \mathbf{u}_{2}\right)+\mathcal{N}\left(\mathbf{u}_{3}, \mathbf{u}_{-2}\right)+\mathcal{N}\left(\mathbf{u}_{-2}, \mathbf{u}_{3}\right)+\ldots}_{\mathcal{N}_{1}}
$$

If, as is often the case, ||$u_{n}|| \sim \epsilon^{|n|}$, then $\mathcal{N}_{1}=O\left(\epsilon^{3}\right)$ may be neglected and RZIF is satisfied: the linear operator $\mathcal{L}_{\bar{U}}$ in (6) has the pure imaginary eigenvalue $i \omega$, corresponding to the frequency of the periodic solution. Hence the RZIF property is satisfied for near-monochromatic oscillations in any system with a quadratic nonlinearity. 


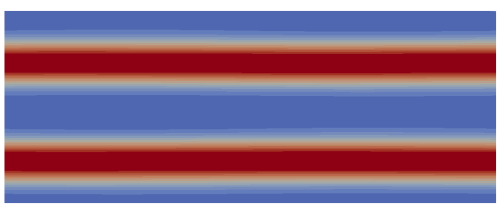

$t=19.33$

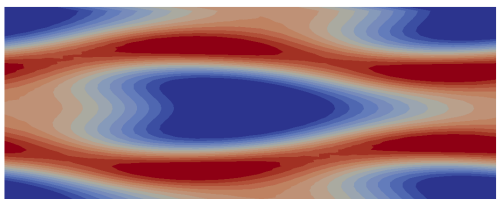

$t=20.86$

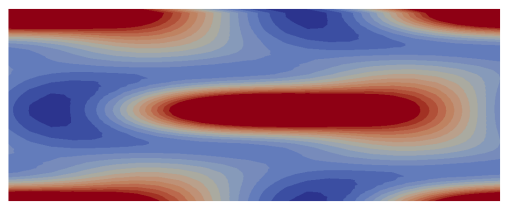

$t=21.08$

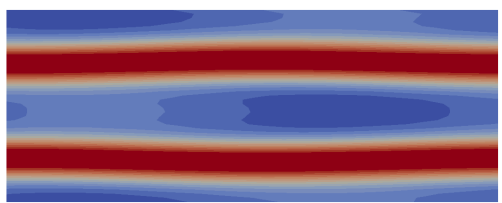

$t=20.33$

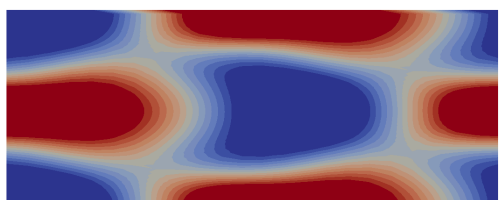

$t=20.91$

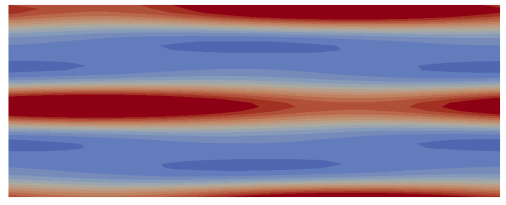

$t=21.49$
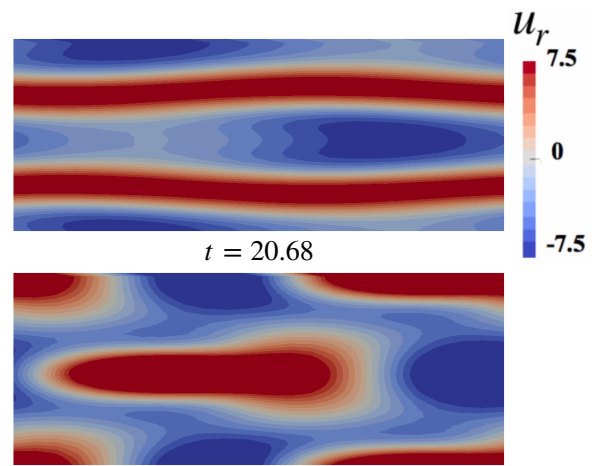

$t=20.98$

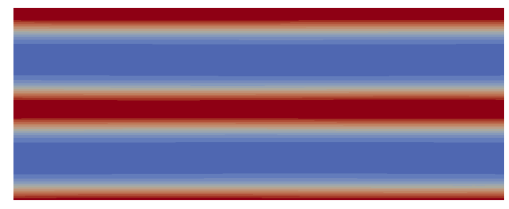

$t=21.83$

FIG. 8. Non-axisymmetric heteroclinic cycle at $R e=-502$. Colors show the radial velocity in a $(\theta, z)$ slice at $r=1.26$. The first (top left) and last (bottom right) visualizations show the saddles which anchor the heteroclinic cycles. These contain two pairs of axisymmetric vortices and differ by an axial phase shift of a single vortex. The visualizations between the two illustrate the excursion or rapid transition between the two saddles, via states which resemble ribbons.

It remains to verify whether these periodic solutions are near-monochromatic. Figure 7 shows the temporal Fourier spectra of the azimuthal velocity for the spirals and the ribbons. For the thermosolutal case of [10], the ratio $\left\|u_{2}|| /\right\| u_{1} \|$ was found to be much higher for the standing waves (greater than about $10^{-1}$ ) than for the traveling wave branch (between $10^{-3}$ and about $10^{-1}$ ), explaining the satisfaction of RZIF for the traveling waves and not for the standing waves. In contrast, for this counter-rotating Taylor-Couette case, figure 7 shows that the temporal spectra for spirals and ribbons are quite similar. The ratio $\left\|u_{2}\right\| /\left\|u_{1}\right\|$ is consistently less than $10^{-2}$ for both flows over the range of our investigation. This is consistent with the fact that RZIF is satisfied for both flows.

\section{HETEROCLINIC ORBITS}

Pinter et al. [18 20] observed that the ribbon branch became unstable and was succeeded by oscillating cross-spirals as $R e$ was increased. We observe this as well, near $R e \approx-525$. As $R e$ is further increased, however, the oscillating cross-spirals are themselves succeeded by a near-heteroclinic orbit, in which the system spends the overwhelming majority of its time in one of two saddles containing two pairs of axisymmetric vortices. The two saddles differ by a quarter of an axial period (one vortex). The system passes from one to the other via rapid non-axisymmetric excursions resembling the ribbon states. Snapshots of the phases of an excursion taken from a heteroclinic cycle at $R e=-502$ are shown in figure 8 .

Figure $9(\mathrm{a}, \mathrm{b}, \mathrm{c})$ shows timeseries from this heteroclinic orbit. Time spent at the two saddles are seen as two plateaus. The approaches to and departures from the saddles are oscillatory; this appears clearly in the enlarged timeseries of figure $9 \mathrm{~b}$, as well as in the spirals of the phase portrait of figure $10 \mathrm{a}$. In the phase portrait, the two saddles are distinguished, and the spiraling approaches to them are approximately perpendicular to one another. In the timeseries of figure 98, the energy in the non-axisymmetric modes is seen to increase and to decrease logarithmically.

As Re is further increased, this regime is succeeded by another type of heteroclinic orbit, visualized in figure 11. Timeseries for this cycle are shown in figure $9(\mathrm{~d}, \mathrm{e}, \mathrm{f})$ for $R e=490$. The two plateaus are the same as for the previous heteroclinic cycle, but the approaches to and departures from the saddles are not oscillatory. These approaches and departures differ from excursion to excursion, as highlighted in the enlargements in figure 9 e. The difference between the excursions is also seen in the phase portraits in figure $10 \mathrm{p}$, which begins with a transient oscillatory phase. The black dots in the phase portrait are equally spaced in time and so accumulate on the saddles. Trajectories are seen approaching and leaving the saddles along different paths at different angles. Turning to the logarithmic energy timeseries of figure 9(f), after an initial transient (corresponding to the oscillatory transient in the phase portrait), the energy in the non-axisymmetric modes becomes and remains very small. Instead, it is the energy in the odd axial wavenumber modes which tracks the heteroclinic cycle. Since the saddles contain two stacked pairs of vortices, their 


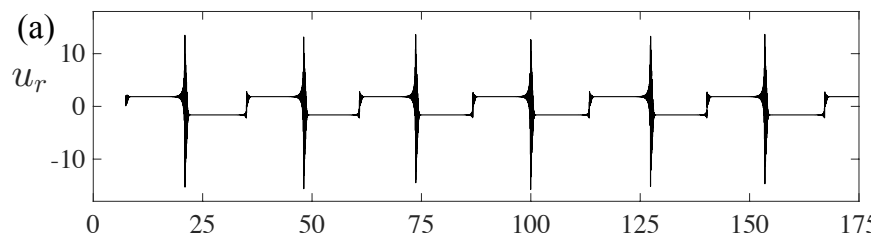

(d)
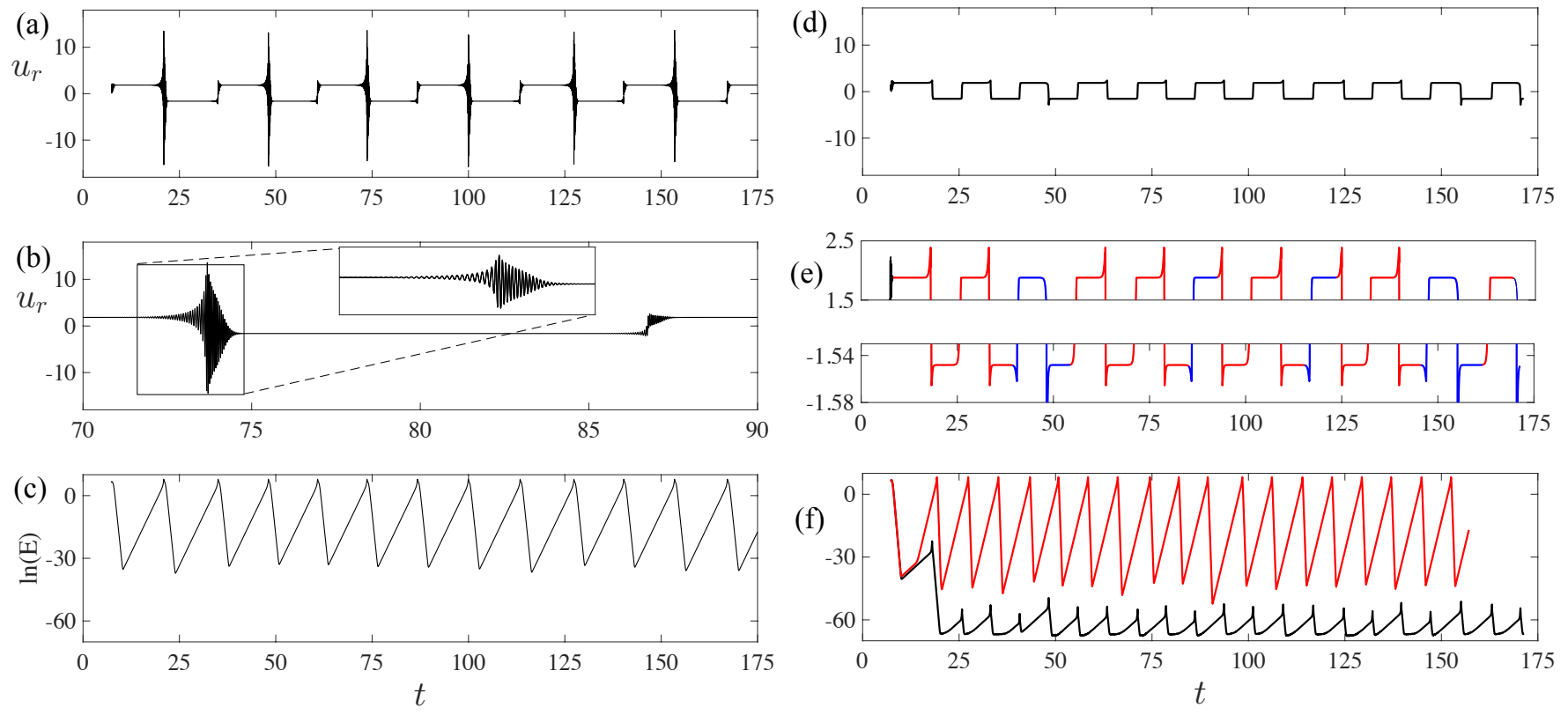

FIG. 9. Timeseries for (a,b,c) oscillatory and non-axisymmetric heteroclinic cycle at $R e=-502$ and for (d,e,f) non-oscillatory and axisymmetric heteroclinic cycle at $R e=-490$. (a,d) Complete timeseries alternating between two plateaus. (b,e) Enlargements of timeseries. b) Enlargement of a single period from a non-axisymmetric cycle, with further enlargement around the excursion. The differences between the growth/decay rates and frequencies during the spiralling approach and departure are clear. e) Enlargements near the peaks of the axisymmetric non-oscillatory excursions, highlighting their variation. (c,f) Logarithmic plot of energy, which grows and decays exponentially. The non-axisymmetric energy is shown in black and, for the axisymmetric heteroclinic cycle in (f), the energy corresponding to axisymmetric but axially antisymmetric modes is shown in red. Both types of heteroclinic cycles have the same plateaus, up to numerical roundoff.
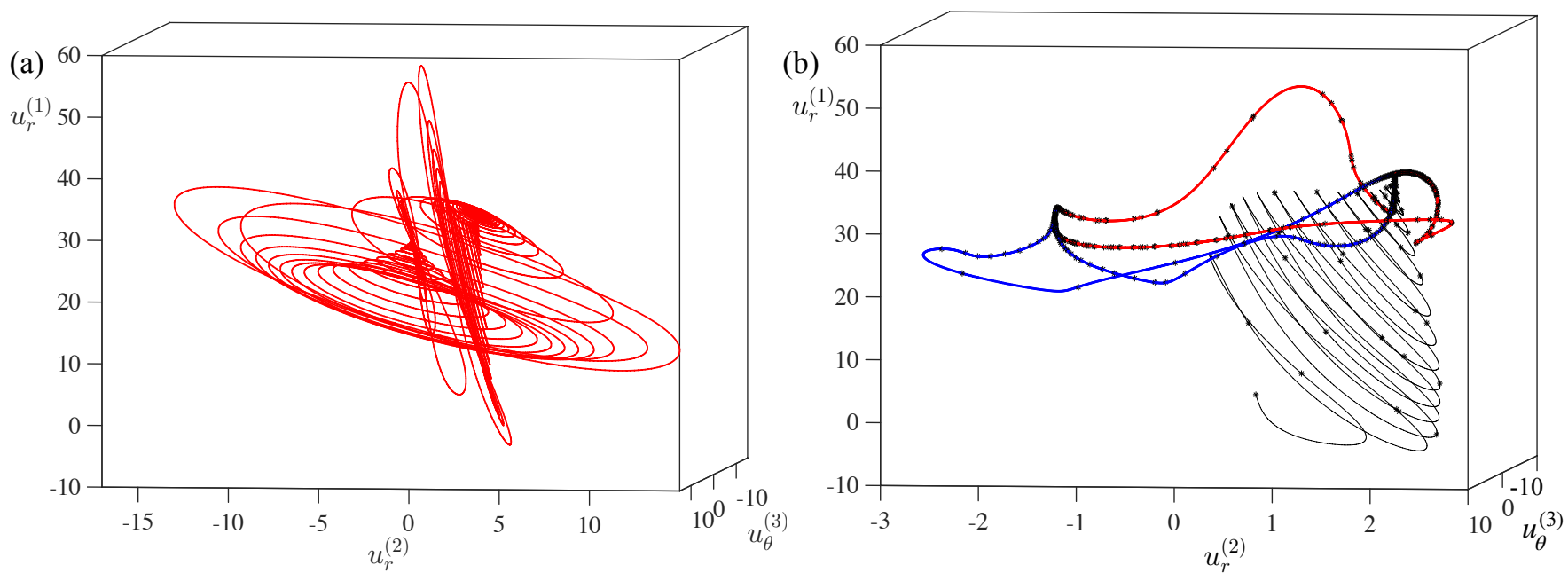

FIG. 10. a) Phase portraits for a) oscillatory heteroclinic cycle at $R e=-502$ and for b) non-oscillatory heteroclinic cycle at $R e=-490$ using $u_{r}(r=1.2, \theta=0, z=0), u_{r}\left(r=1.2, \theta=\pi / 3, z=\lambda_{z} / 3\right)$, and $u_{\theta}\left(r=1.2, \theta=2 \pi / 3, z=2 \lambda_{z} / 3\right)$ corresponding to timeseries in figure 9. The trajectory in the oscillatory heteroclinic cycle spirals inwards along one curved surface and outwards along a perpendicular curved surface. Non-oscillatory phase portrait begins with a spiralling transient, then follows different paths into and out of the saddles. The color coding (blue and red) of these paths matches that in the timeseries, where can be seen subtle differences between the excursions. The saddles for the two cycles are the same; note the different scale for $u_{r}^{(2)}$ between (a) and (b). 


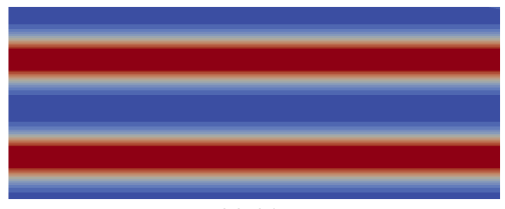

$t=32.39$

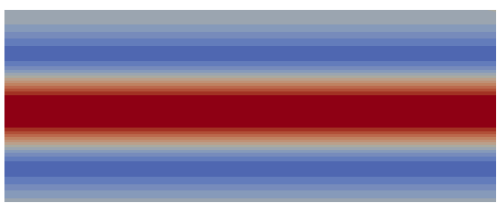

$t=33.13$

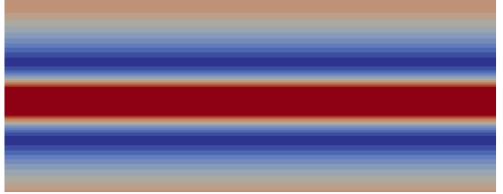

$t=33.19$

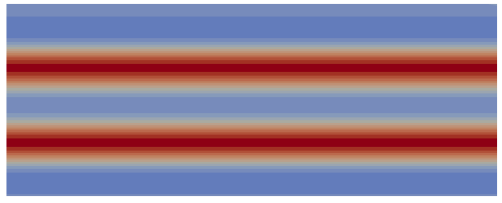

$t=33.09$

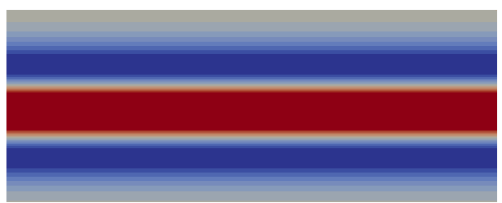

$t=33.14$

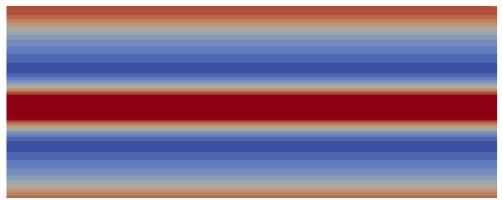

$t=33.24$
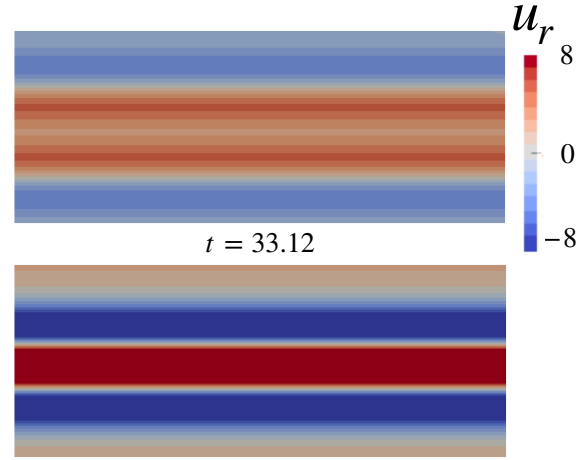

$t=33.16$

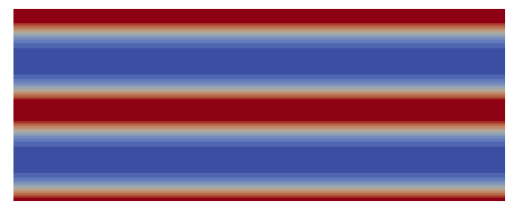

$t=33.59$

FIG. 11. Axisymmetric heteroclinic cycle at $R e=-490$. Colors show the radial velocity in a $(\theta, z)$ slice at $r=1.26$. The first (top left) and last (bottom right) visualizations show the saddles which anchor the heteroclinic cycles. These contain two pairs of axisymmetric vortices and differ by an axial phase shift of a single vortex. For this heteroclinic cycle, the states remain axisymmetric throughout the rapid transition between the two saddles.

axial Fourier spectrum contain only even multiples of the fundamental wavenumber $2 \pi / \lambda_{z}$. Excursions are manifested by the logarithmic increase and decrease of the energy contained in odd multiples of $2 \pi / \lambda_{z}$ in the axial spectrum. The variation from cycle to cycle is also seen in the logarithmic timeseries.

The axisymmetric heteroclinic cycle is very similar to that observed in the 1:2 mode-interaction [26, 27]. An erratic alternation between a few different types of excursions is also seen in the cycles of [27. As explained in these references, this cycle is a consequence of the interaction of axial modes with wavenumbers $2 \pi / \lambda_{z}$ and $4 \pi / \lambda_{z}$. Furthermore, the heteroclinic orbits can be characterized quantitatively by calculating the rates of approach and departure from the saddles which anchor the heteroclinic orbits. These are the two leading eigenvalues (in the axisymmetric subspace) of the saddles and are seen as the slopes of the black curve in the logarithmic timeseries in figure 9F. We plot these as a function of $R e$ in figure $12 \mathrm{~b}$. The existence of the heteroclinic cycle requires eigenvalues of opposite signs; thus, the axisymmetric cycle exists for $R e>-502$, where one of the leading eigenvalues becomes positive while the other remains negative. Stability of the cycle requires that the rate of departure be no greater than the rate of approach, i.e. the absolute value of the negative eigenvalue must exceed the positive eigenvalue. This occurs for $\operatorname{Re}<-482$. Hence the axisymmetric cycle exists and is stable within this axisymmetric subspace in the range $R e \in[-502,-482]$.

The theory in [26, 27] does not concern oscillatory heteroclinic cycles or complex eigenmodes. However, assuming that the analysis of the oscillatory cycles is similar to that of the non-oscillatory cycles, we plot the slopes (or real parts of the eigenvalues) seen in the logarithmic timeseries of figure $9 \mathrm{k}$ as a function of $R e$. We also plot the frequencies associated with these eigenvalues in figure $13 \mathrm{k}$, associated with the oscillations in the timeseries in figure $9 \mathrm{a}, \mathrm{b}$ and the spiraling in and out in the phase portraits of figure $10 \mathrm{a}$. Using the same criteria as for the non-oscillatory cycles, figure 12 a shows that the oscillatory heteroclinic cycle exists and is stable within the subspace of these eigendirections for $R e \in[-522,-476]$.

The theory in [26, 27] also does not address the relative stability of two kinds of heteroclinic cycles. The transition that we observe when $R e$ is reduced from -502 to -490 implies that the non-axisymmetric heteroclinic cycles is unstable to the axisymmetric one at this value of $R e$. In fact, we observe non-axisymmetric heteroclinic cycles for $R e<-496$ and axisymmetric cycles for $R e>-496$, separated by the point at which axisymmetric and nonaxisymmetric departure rates cross, as seen in figure 13b. This supports the idea that the cycle whose departure rate from the saddles is smaller is unstable to the cycle whose departure rate is greater [28]. Combining all of the criteria, the stability range of the non-axisymmetric cycle is $R e \in[-522,-496]$, while that of the axisymmetric cycle is $R e \in[-496,-482]$. 

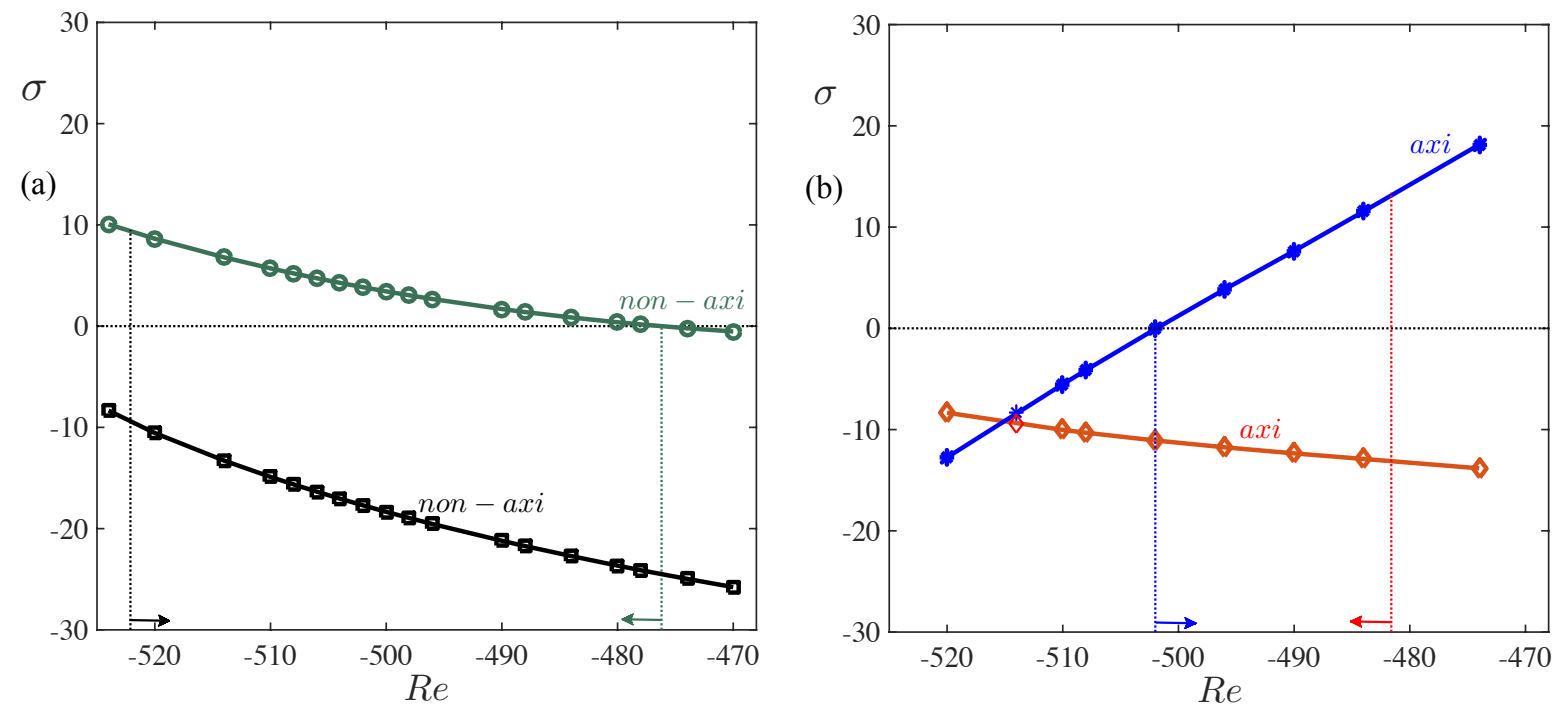

FIG. 12. Rate of approach to and departure from saddles as a function of Re. These are the slopes of the logarithmic plots of figure 9 and also the real parts of the leading eigenvalues. a) Non-axisymmetric oscillatory heteroclinic cycle. The cycle exists when the eigenvalues are of opposite sign, here for $R e<-476$. The cycle is stable within the space of these eigenmodes when the rate of approach exceeds the rate of departure, here for $R e>-522$. b) Axisymmetric non-ocillatory heteroclinic cycle. The cycle exists when the eigenvalues are of opposite sign, here for $R e>-502$. The cycle is stable within the space of these eigenmodes when the rate of approach exceeds the rate of departure, here for $R e<-482$.
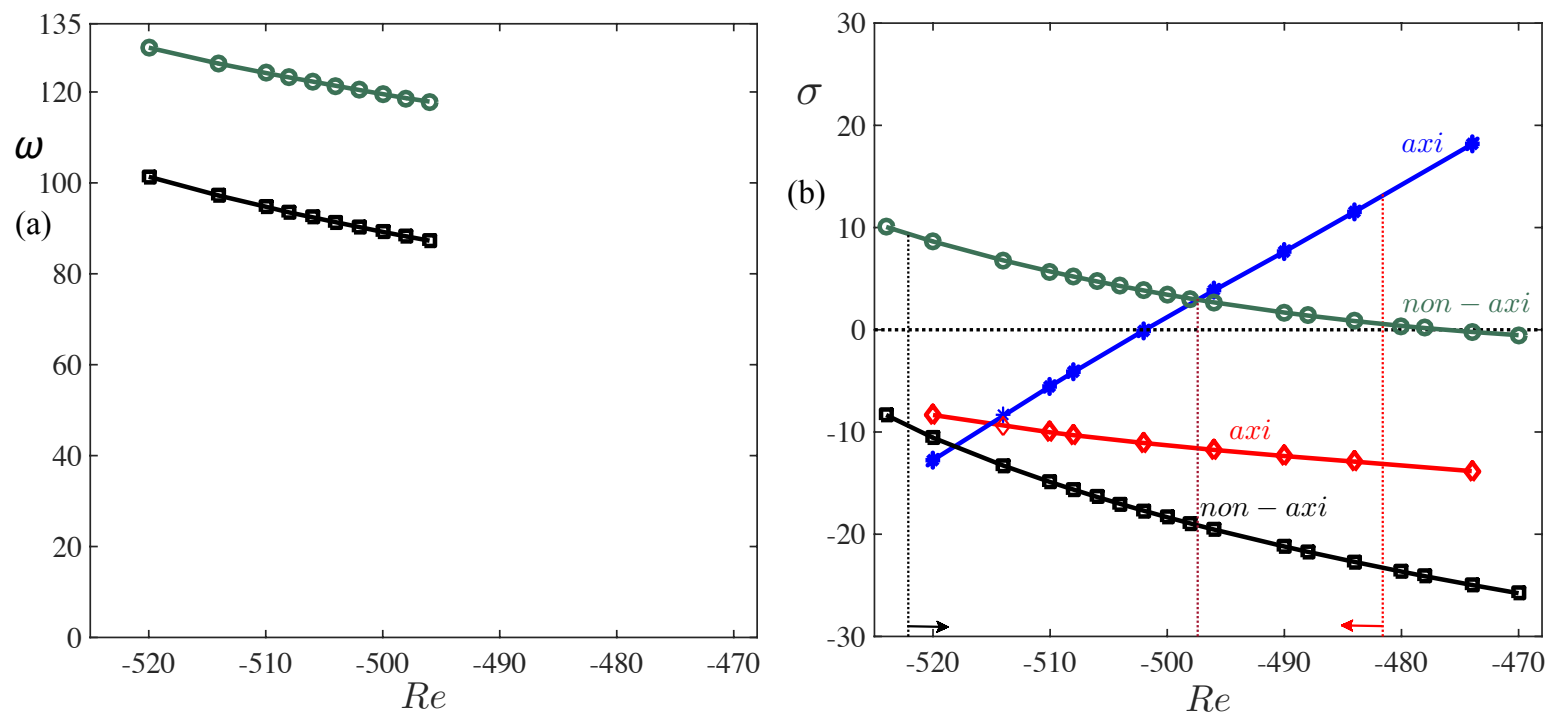

FIG. 13. a) Imaginary parts of eigenvalues involved in non-axisymmetric oscillatory heteroclinic cycle. b) Real parts of eigenvalues involved in both heteroclinic cycles, axisymmetric and non-axisymmetric. Non-axisymmetric cycles are observed for $R e<-496$, when the largest eigenvalue (departure rate, dark green circles) corresponds to a non-axisymmetric eigenpair. Axisymmetric cycles are observed for $R e>-496$, when the largest eigenvalue (blue stars) corresponds to an axisymmetric eigenvector. The other endpoints of the stability ranges, shown as the black rightwards and red leftwards arrows, involve the next-to-largest eigenvalues (rates of approach, red diamonds and black squares); see figure 12 and its caption. Summarizing, the stability range of the non-axisymmetric cycle is $R e \in[-522,-496]$, while that of the axisymmetric cycle is $R e \in[-496,-482]$, which matches our observations. 


\section{DISCUSSION}

The problem of characterizing the nonlinear frequency of a periodic flow away from the threshold has led to one proposed solution: when a linear stability analysis is carried out about the temporal mean, an eigenvalue is obtained whose Real part is Zero and whose Imaginary part is the nonlinear Frequency. This RZIF property has heretofore been studied for the cylinder wake, an open cavity, and thermosolutal convection. It is strongly satisfied for the cylinder wake and the traveling waves of thermosolutal convection with oppositely directed density gradients, weakly satisfied for the open cavity, and not at all satisfied for the standing waves of thermosolutal convection. Although the RZIF property is a natural outcome of a near-monochromatic temporal spectrum, it is not entirely clear when this occurs.

We have investigated RZIF in another configuration, counter-rotating Taylor-Couette flow, which, like the thermosolutal convective case, has a Hopf bifurcation leading to branches of traveling and standing waves, here manifested as spirals and ribbons. In the thermosolutal case, the traveling waves display the RZIF property and have a temporal Fourier spectrum which is highly peaked, while the standing waves do not. Here, both the spirals and the ribbons display the RZIF property and both have temporal spectra which are equally peaked. However, the ribbons are standing waves only in the axial direction and are traveling waves in the azimuthal direction. This may be the cause of the difference between the Taylor-Couette and thermosolutal cases. The search for another counter-example to RZIF, especially in a purely hydrodynamic flow without additional fields, remains open.

During the course of our investigation, we discovered two heteroclinic cycles, one non-axisymmetric and the other axisymmetric. Both cycles are anchored by the same saddles: axisymmetric states containing two axially stacked pairs of vortices. The axisymmetric heteroclinic cycle is a manifestation of the classic 1:2 mode interaction, here the interaction between axisymmetric states with one and two pairs of vortices. The non-axisymmetric heteroclinic cycle is not of this type, since the approach to and from the saddles is oscillatory via transients resembling ribbons. However, it is anchored by the same axisymmetric saddles. The existence of two qualitatively different heteroclinic cycles connecting the same saddles is an unusual and intriguing feature. These cycles will be studied in more detail in a later investigation.

\section{ACKNOWLEDGMENTS}

This work was performed in part using high performance computing resources provided by the Institut du Developpement et des Ressources en Informatique Scientifique (IDRIS) of the Centre National de la Recherche Scientifique (CNRS), coordinated by GENCI (Grand Equipement National de Calcul Intensif) through grant A0042A01119.

[1] D. Barkley, "Linear analysis of the cylinder wake mean flow," Europhys. Lett. 75, 750 (2006).

$[2]$ B. Pier, "On the frequency selection of finite-amplitude vortex shedding in the cylinder wake," J. Fluid Mech. 458, 407-417 (2002).

[3] B. R. Noack, K. Afanasiev, M. Morzyński, G. Tadmor, and F. Thiele, "A hierarchy of low-dimensional models for the transient and post-transient cylinder wake," J. Fluid Mech. 497, 335-363 (2003).

[4] S. Mittal, "Global linear stability analysis of time-averaged flows," Int. J. Numer. Meth. Fluids 58, 111-118 (2008).

[5] V. Mantič-Lugo, Cristó.bal Arratia, and François Gallaire, "Self-consistent mean flow description of the nonlinear saturation of the vortex shedding in the cylinder wake," Phys. Rev. Lett. 113, 084501 (2014).

[6] V. Mantič-Lugo, C. Arratia, and F. Gallaire, "A self-consistent model for the saturation dynamics of the vortex shedding around the mean flow in the unstable cylinder wake," Phys. Fluids 27, 074103 (2015).

[7] D. Sipp and A. Lebedev, "Global stability of base and mean flows: a general approach and its applications to cylinder and open cavity flows," J. Fluid Mech. 593, 333-358 (2007).

[8] P. Meliga, "Harmonics generation and the mechanics of saturation in flow over an open cavity: a second-order self-consistent description," J. Fluid Mech. 826, 503-521 (2017).

[9] Y. Bengana, J.-C. Loiseau, J.-C. Robinet, and L.S. Tuckerman, "Bifurcation analysis and frequency prediction for open cavity flow," submitted to J. Fluid. Mech..

[10] S. E. Turton, L. S. Tuckerman, and D. Barkley, "Prediction of frequencies in thermosolutal convection from mean flows," Phys. Rev. E 91, 043009 (2015).

[11] E. Knobloch, "Oscillatory convection in binary mixtures," Phys. Rev. A 34, 1538 (1986).

[12] E. R. Krueger, A. Gross, and R. C. Di Prima, "On the relative importance of Taylor-vortex and non-axisymmetric modes in flow between rotating cylinders," J. Fluid Mech. 24, 521-538 (1966).

[13] W. F. Langford, R. Tagg, E. J. Kostelich, H. L. Swinney, and M. Golubitsky, "Primary instabilities and bicriticality in flow between counter-rotating cylinders," Phys. Fluids 31, 776-785 (1988). 
[14] J. Antonijoan, F. Marques, and J. Sanchez, "Non-linear spirals in the Taylor-Couette problem," Phys. Fluids 10, 829-838 (1998).

[15] Y. Demay and G. Iooss, "Calcul des solutions bifurquées pour le problème de Couette-Taylor avec les 2 cylindres en rotation," J. Mec. Théor. Appl., 193-216 (1984), special issue.

[16] R. Tagg, W. S. Edwards, H. L. Swinney, and P. S. Marcus, "Nonlinear standing waves in Couette-Taylor flow," Phys. Rev. A 39, 3734 (1989).

[17] P. Chossat and G. Iooss, The Couette-Taylor Problem (Springer, 2012).

[18] A. Pinter, M. Lücke, and C. Hoffmann, "Competition between traveling fluid waves of left and right spiral vortices and their different amplitude combinations," Phys. Rev. Lett. 96, 044506 (2006).

[19] A. Pinter, M. Lücke, and C. Hoffmann, "Bifurcation of standing waves into a pair of oppositely traveling waves with oscillating amplitudes caused by a three-mode interaction," Phys. Rev. E 78, 015304(R) (2008).

[20] A. Pinter, M. Lücke, and C. Hoffmann, "Wave-number dependence of the transitions between traveling and standing vortex waves and their mixed states in the Taylor-Couette system," Phys. Rev. E 78, 017303 (2008).

[21] C. Hoffmann, S. Altmeyer, A. Pinter, and M. Lücke, "Transitions between Taylor vortices and spirals via wavy Taylor vortices and wavy spirals," New J. Phys. 11, 053002 (2009).

[22] S. Altmeyer and C. Hoffmann, "Secondary bifurcation of mixed-cross-spirals connecting travelling wave solutions," New J. Phys. 12, 113035 (2010)

[23] K. Deguchi and S. Altmeyer, "Fully nonlinear mode competitions of nearly bicritical spiral or Taylor vortices in TaylorCouette flow," Phys. Rev. E 87, 043017 (2013).

[24] R. E. Ecke, F. Zhong, and E. Knobloch, "Hopf bifurcation with broken reflection symmetry in rotating Rayleigh-Bénard convection," Europhys. Lett. 19, 177 (1992).

[25] T. Dessup, L. S. Tuckerman, J. E. Wesfreid, D. Barkley, and A. Willis, "The self-sustaining process in Taylor-Couette flow," Phys. Rev. Fluids 3, 123902 (2018).

[26] D. Armbruster, J. Guckenheimer, and P. Holmes, "Heteroclinic cycles and modulated travelling waves in systems with O(2) symmetry," Physica D 29, 257-282 (1988).

[27] C. Nore, L. S. Tuckerman, O. Daube, and S. Xin, "The 1:2 mode interaction in exactly counter-rotating von Kármán swirling flow," J. Fluid Mech. 477, 51-88 (2003).

[28] P. Ashwin and P. Chossat, "Attractors for robust heteroclinic cycles with continua of connections," J. Nonlinear Sci. 8, 103-129 (1998). 